\title{
Evaluación de la utilización de Cratylia argentea como suplemento en dietas para pollos de engorde
}

\section{Evaluation of the use of Cratylia argentea as a supplement in diets for broilers}

\author{
Silva Higuera Andrea Carolina ${ }^{1}$, Zuluaga Hoyos Ana María ${ }^{1}$ y \\ Roa Vega María Ligia² \\ ${ }^{1}$ Estudiantes de Medicina Veterinaria y Zootecnia, Curso de profundización \\ "Sistemas silvopastoriles y de alimentación en el trópico" \\ 2Zootecnista, Esp., MSc., Docente Universidad de los Llanos \\ mroa@unillanos.edu.co
}

Recibido 27 de Febrero 2013, Aceptado 02 de Abril 2013

\section{RESUMEN}

Las leguminosas forrajeras se pueden tener en cuenta para reemplazar parte de la proteína requerida en las dietas para aves, con el fin de disminuir los costos en las producciones familiares. El propósito de la presente investigación fue determinar las ventajas de incluir Cratylia argentea en diferentes porcentajes, en dietas para la alimentación de pollos de engorde. El Trabajo se realizó con 36 pollos de engorde (Arbor acres) de 15 días de edad con peso promedio de $450 \pm 20 \mathrm{~g}$, se les ofreció dietas balanceadas: isoprotéicas $(21 \%)$ e isoenergéticas $(2.900 \mathrm{Kcal}$ de EM (energía metabolizable) / kg), a las cuales se les incluyó diferentes niveles de harina de Cratylia argentea $(\mathrm{HC})$ de la siguiente manera: Testigo $\mathrm{T} 0=0 \%, \mathrm{~T} 1=$ $5 \%, \mathrm{~T} 2=10 \%$ y T3 $=15 \%$. Los principales ingredientes utilizados fueron harina de carne, torta de soya, harina de arroz y maíz. Durante cinco días se hizo un acostumbramiento de las aves a estas dietas que les fueron ofrecidas, cada cinco días se pesaron las aves para determinar su ganancia. Para medir el valor biológico (VB) de la proteína, se recolectaron las excretas durante tres días las cuales fueron analizadas en el Laboratorio de Nutrición Animal, proceso igual se realizó con las dietas ofrecidas y la HC. El diseño estadístico que se utilizó fue un diseño completamente al azar: cuatro tratamientos, tres repeticiones y tres unidades experimentales. Los pollos que obtuvieron una mejor ganancia de peso 
total $(P<0,05)$ fueron los alimentados con las dietas T0 y T2, al igual que el peso total de la canal. El porcentaje del valor biológico de la proteína, fue similar para los cuatro tratamientos, aunque la fibra detergente neutro que contiene la Cratilya, afectó negativamente su digestibilidad. De acuerdo a estos resultados se deduce que la inclusión del $5 \%$ de $\mathrm{HC}$ en dietas para pollos de engorde no afecta las variables evaluadas.

Palabras clave: Leguminosa forrajera, alimentación, aves de engorde.

\begin{abstract}
Forage legumes can consider to replace part of the protein required in poultry diets in order to reduce costs in family economy. The purpose of this research was to determine the advantages of Cratylia argentea include it in different levels, in diets for broiler feed. This work was carried out with 36 broilers (Arbor acres) 15 days of old with an average weight of $450 \pm 20 \mathrm{~g}$, they received balanced diets: the same protein (21\%) and caloric content (2,900 kcal ME (metabolizable energy) / kg), to which different levels are included Cratylia argentea flour $(\mathrm{HC})$ as follows: Control $\mathrm{T} 0=0 \%, \mathrm{~T} 1=5 \%, \mathrm{~T} 2=10 \%$, and $\mathrm{T} 3=15 \%$. The main ingredients used were flour beef, soybean meal, rice flour and corn. For five days there was a habituation of birds to these diets that were offered, every five days the birds were weighed to determine their weight gain. To measure the biological value (BV) protein, excreta were collected for three days which were analyzed in the Laboratory of Animal Nutrition; the same process was carried out with diets offered and HC. The statistical design used was completely randomized design, four treatments, three replicates and three experimental units. Chickens were better total weight gain $(P<0.05)$ were fed diets T0 and T2, as the total weight of the beef carcass. The percentage of the biological value of the protein was similar for all four treatments, although neutral detergent fiber containing Cratilya negatively affected digestibility. According to these results it follows that the inclusion of $5 \%$ of $\mathrm{HC}$ in broiler diets does not affect variables.
\end{abstract}

Keywords: Forage legume, feeding, broilers. 


\section{INTRODUCCIÓN}

Los sistemas de producción animal en Colombia tienen como eje central, el manejo de la alimentación, para el mejoramiento de la calidad de los productos como carne, leche y huevos. Este factor ha contribuido en las últimas décadas a la búsqueda de nuevas alternativas que van desde alimentos concentrados que proporcionan al animal el balance necesario de nutrientes para el desarrollo de sus procesos fisiológicos normales, hasta sales mineralizadas en el caso de rumiantes, las cuales proporcionan los microelementos suficientes para cubrir sus necesidades nutricionales. Sin embargo, aunque existen estas opciones, dado que las materias primas con las cuales se encuentran elaboradas en su mayoría son importadas, generan un valor adicional al producto utilizado (Miles et al., 2004).

La utilización de leguminosas forrajeras, puede ser una alternativa para reemplazar, el uso de concentrado en producciones avícolas familiares, y de esta forma mantener el crecimiento de las aves, además de bajar los costos. El arbusto Cratylia argentea, es una leguminosa que se ha venido integrado en los sistemas de producción animal en el trópico. Esta especie ofrece buenos rendimientos en materia seca comparándola con las forrajeras herbáceas, y además tolera mejor las condiciones adversas, teniendo la capacidad de rebrotar y de esta forma ofrecer forraje de buena calidad en condiciones de alto estrés ambiental (Abreu et al., 2003).

Actualmente la búsqueda de los nuevos elementos para la alimentación animal tiene como objetivo disminuir los costos de producción al mismo tiempo que los indicadores de producción se aumenten, como es el caso del uso de plantas de uso forrajero que a la vez contribuyen a generar alimentos de consumo humano sin aditivos artificiales (Seddon, 2004).

El género Cratylia pertenece a la familia Leguminosae, subfamilia Papilionoideae, tribu Phaseoleae y subtribu Diocleinae; crece en forma de arbusto de 1.5 a $3.0 \mathrm{~m}$ de altura o en forma de lianas volubles. Las hojas son trifoliadas y estipuladas, los folíolos son membranosos o coriáceos con los dos laterales ligeramente 
asimétricos; la inflorescencia es un pseudoracimo nodoso con 6 a 9 flores por nodosidad; las flores varían en tamaños de 1.5 a $3.0 \mathrm{~cm}$ con pétalos de color lila y el fruto es una legumbre dehiscente que contiene de 4 a 8 semillas en forma lenticular, circular o elíptica (Lascano et al., 2002).

Cratylia argentea es un arbusto nativo de la Amazonia, de la parte central de Brasil y de áreas de Perú, Bolivia y Argentina. Se caracteriza por su amplia adaptación a zonas bajas tropicales con sequías hasta de seis meses en suelos ácidos de baja fertilidad del tipo ultisol y oxisol. Bajo estas condiciones produce buenos rendimientos de forraje bajo corte y tiene la capacidad de rebrotar durante el período seco debido a un desarrollo radicular vigoroso. Por otra parte, produce abundante semilla y su establecimiento es relativamente rápido cuando las condiciones son adecuadas (Rincón, 2005).

En el género Cratylia se han reconocido cinco especies diferentes, las cuales son: C. bahiensis C. hypargyrea, C. intermedia, C.mollis y C. argentea. La diferenciación entre especies se ha logrado con base a características morfológicas vegetativas y la ubicación geográfica de éstas, debido a que no existen hasta la fecha estudios reproductivos ni de hibridación que permitan una clasificación de especie basada en marcadores biológicos (Lascano et al., 2002).

C. argentea, es la especie de más amplia distribución en Sur América es de tipo arbustivo en formaciones vegetales abiertas, pero puede convertirse en liana de tipo voluble cuando está asociada a plantas de porte mayor. La especie se ramifica desde la base del tallo y se reportan hasta 11 ramas en plantas de $1.5 \mathrm{a}$ $3.0 \mathrm{~m}$ de altura. Las hojas tienen consistencia papirácea con abundante pubescencia en el envés (Jimenez y Tovar, 2003).

En Colombia las características del suelo de la Orinoquía permiten que la $C$. argentea sea una leguminosa óptima para ser usada en alimentación animal. Se ha observado que su consumo es mejor cuando se suministra como forraje maduro o cuando se orea o seca al sol durante 24 horas en comparación cuando se le suministra en estado fresco (Lascano y Plazas, 2003). 
Los contenidos promedios de proteína de $C$. argentea en la Orinoquía son de $21,7 \%$ lo que la convierte en una buena oportunidad de utilización debido a que presenta una buena producción de hoja verde durante la época seca en comparación con las gramíneas, en otros estudios con $C$. argentea se dan resultados de proteína cruda de $23,5 \%$, digestibilidad in vitro de materia seca de $48 \%$ y reportan bajos niveles de taninos, lo cual la convierten en una excelente fuente de proteína para ser usada en la alimentación animal (Romero y González, 2001).

Las dietas para pollos de engorde actualmente están siendo revisadas, debido a su contenido de aditivos no nutricionales, que están afectando la salud humana, por lo tanto, ha sido necesario replantear alternativas para proponer dietas que contengan ingredientes de excelente calidad de manera orgánica, dando cumplimiento a normas de bioseguridad y manejo, vacunación, selección genética, entre otros (Reyes, 2001). Las leguminosas forrajeras se pueden tener en cuenta para reemplazar parte de la proteína requerida en la dieta de la dieta.

En un trabajo con pollos de engorde de un día de nacidos siendo alimentados hasta los 20 días de edad con concentrado de iniciación, luego se les ofreció dietas balanceadas las cuales fueron isoprotéicas (21\%) e isoenergéticas $(2.900$ Kcal de EM (energía metabolizable) / kg), a las que se les incluyeron diferentes niveles de harina de cayeno (Hibiscus rosa-sinensis): 0, 5, 8 y 12\%, se concluye que la harina de cayeno puede reemplazar parte de la proteína en las dietas para pollos de engorde hasta en un $12 \%$, sin que se afecte negativamente el consumo de alimento, aumento de peso, conversión, valor biológico de la proteína y digestibilidad de la fibra detergente neutro, es importante señalar que las canales de las aves alimentadas con cayeno, presentaron una coloración amarilla y mejor gustosidad en comparación con el testigo (Roa, 2000).

En dos experimentos realizados en Villavicencio se observó el comportamiento de pollos de engorde reemplazando en las dietas la fuente de proteína tradicional con harina de nacedero Trichanthera gigantea y harina de poró Erythrina poeppigiana en niveles de: 0, 5, 10 y 15\%. En el caso del nacedero se observó que cuando se 
iban incrementando sus niveles el consumo de alimento decreció, lo mismo pasó con el valor biológico de la proteína; la ganancia de peso, y la conversión fueron similares para el testigo (62.9 gramos/día/ave y 2.5 ) y $5 \%$ de harina de nacedero (65.0 gramos/día/ave y 2.3). En el otro experimento con harina de poro, que es una leguminosa, el consumo de alimento y el valor biológico de la proteína, no se afectaron cuando se incrementaron sus niveles, mientras que el aumento de peso fue menor para el 12\% de reemplazo (50.2 gramos/día/ave) (Roa, 2010).

Con lo expuesto anteriormente, el propósito del presente trabajo fue el de aprender sobre el manejo de $C$. argentea para ser suministrada a pollos de engorde con el fin de reemplazar el concentrado comercial, lo cual puede tener un impacto económico potencial en producciones familiares sin detrimento del crecimiento de los pollos y por tanto no afectar la producción de su carne, Adicionalmente se espera que las dietas sean en un sentido orgánico que busca beneficiar no solo la salud del animal, sino que también la de consumidor de carne de pollo.

\section{METODOLOGÍA}

Este proyecto de aula se realizó en la sede Barcelona, de la Universidad de los Llanos en la ciudad de Villavicencio en el departamento del Meta, región de la Orinoquia, zona de piedemonte, con una temperatura media: $27^{\circ} \mathrm{C}$, temperatura máxima: $33.3^{\circ} \mathrm{C}$, temperatura mínima: $18.8^{\circ} \mathrm{C}$, altitud de 465 m.s.n.m. y una precipitación anual entre 1900 y $2300 \mathrm{~mm}$.

El trabajo se realizó con 36 pollos de engorde (línea Arbor acres), de 15 días de edad con peso promedio de $450 \pm 20 \mathrm{~g}$, a los que se les ofreció dietas balanceadas las cuales fueron isoprotéicas $(21 \%)$ e isoenergéticas $(2.900 \mathrm{Kcal}$ de EM (energía metabolizable) / kg), a las cuales se les incluyó diferentes niveles de harina de Cratylia argentea $(\mathrm{HC})$ de la siguiente manera: Testigo $\mathrm{T} 0=0 \%, \mathrm{~T} 1=$ $5 \%, \mathrm{~T} 2=10 \%$ y T3 $=15 \%$. En la Tabla 1 se presentan los ingredientes utilizados en los tratamientos hasta los 40 días. Durante cinco días se hizo una adaptación de las aves a estas dietas, con el agua fueron ofrecidas a voluntad, registrándose 
su consumo diario, cada cinco días se pesaron las aves para determinar su ganancia. Para medir el valor biológico (VB) de la proteína, se recolectaron las excretas durante tres días las cuales fueron analizadas en el Laboratorio de Nutrición Animal, proceso igual se realizó con las dietas ofrecidas y la harina de cratilia (Tabla 2).

Tabla 1. Ingredientes utilizados en dietas con Cratilya argentea en pollos de engorde entre 15 - 40 días de edad

\begin{tabular}{lcccc}
\hline \multirow{1}{*}{\multicolumn{1}{c}{ Ingredientes }} & \multicolumn{5}{c}{ Tratamientos } \\
\cline { 2 - 5 } & T0 & T1 & T2 & T3 \\
\hline Cratilya & 0.0 & 5.0 & 10.0 & 15.0 \\
Harina de Carne & 12.0 & 10.0 & 8.0 & 5.0 \\
Torta de Soya & 17.0 & 17.0 & 17.0 & 17.0 \\
Harina de Arroz & 20.0 & 19.3 & 18.1 & 17.0 \\
Sorgo & 20.0 & 20.0 & 20.0 & 20.0 \\
Maíz & 21.0 & 20.0 & 20.0 & 20.0 \\
Melaza & 5.0 & 3.0 & 3.0 & 3.0 \\
Aceite de Palma & 2.8 & 3.4 & 3.4 & 3.4 \\
Otros Nutrientes & 2.2 & 2.3 & 2.5 & 2.6 \\
$\quad$ Total & 100.0 & 100.0 & 100.0 & 100.0 \\
\hline Harina de Cratilya argentea $(\mathrm{HC})$. Testigo (T0) $=0 \%$ de HC, T1 $=5 \%$ de HC, T2 $=10 \%$ de HC y T3 \\
$=15 \%$ de HC.
\end{tabular}

Tabla 2. Composición nutricional de la Cratilya argentea y los tratamientos utilizados en pollos de engorde

\begin{tabular}{|c|c|c|c|c|c|}
\hline \multirow{2}{*}{ Nutrientes (\%) } & \multirow{2}{*}{ Cratilya } & \multicolumn{4}{|c|}{ Tratamientos } \\
\hline & & TO & T1 & T2 & T3 \\
\hline Proteína & 17.2 & 21,54 & 21,31 & 20,97 & 20,82 \\
\hline Grasa & 2.4 & 4,5 & 5,1 & 5,5 & 5,8 \\
\hline Fibra cruda & 22.5 & 5 & 5,9 & 6,3 & 7,1 \\
\hline Extracto no nitrogenado & 39,60 & 56,62 & 48,28 & 46,42 & 45,08 \\
\hline Cenizas & 8.3 & 7,34 & 9,41 & 10,81 & 11,2 \\
\hline Fibra detergente Neutro & 47.0 & 24.01 & 26.09 & 28.31 & 30.56 \\
\hline EM (Kcal/kg) & 2371.60 & 2910.50 & 2901.24 & 2893.20 & 2890,02 \\
\hline $\begin{array}{l}\text { Harina de Cratilya argente } \\
\text { T3= } 15 \% \text { de HCA). }\end{array}$ & ). Testigo ( & ) $=0 \%$ de $\mathrm{r}$ & $\mathrm{T} 1=5 \% \mathrm{C}$ & $\mathrm{HC}, \mathrm{T} 2=$ & 6 de HCA y \\
\hline
\end{tabular}


evaluó: aumento de peso, consumo de alimento, conversión, valor biológico (VB) de la proteína, digestibilidad de la fibra detergente neutro (FDN) y rendimiento en canal, se aplicaron las pruebas de medias Tukey.

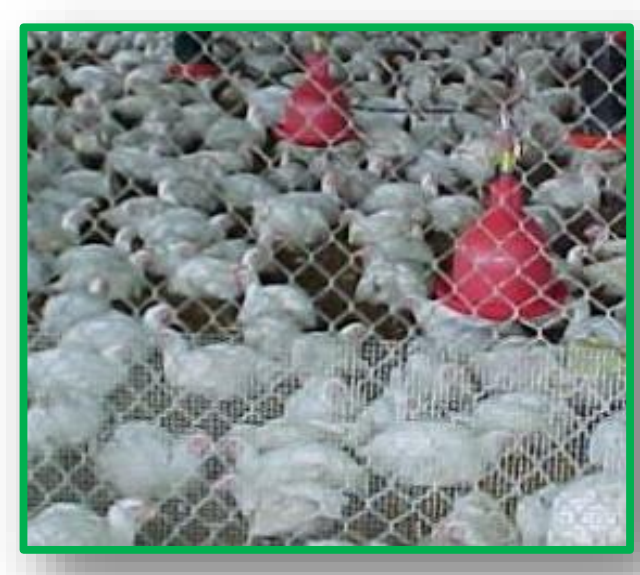

Fotografía 1. El reemplazo de la proteína con harina de leguminosas forrajeras, como la Cratylia, puede ser una alternativa para reducir costos en pequeñas producciones de pollos de enaorde

\section{RESULTADOS Y DISCUSIÓN}

En la composición de la Cratylia y de los tratamientos suministrados a los pollos (Tabla 2), se muestra que la proteína de esta leguminosa $(17,2 \%)$, tiene $4 \%$ menos que los requerimientos de los pollos, que es de $21 \%$, lo cual es suficiente argumento para incluirla en las dietas para no rumiantes, aunque presenta un alto contenido de fibra detergente neutro (47\%), lo que es una desventaja, debido a que los pollos no tienen buena capacidad de degradar la fibra y aprovecharla como lo hacen los rumiantes. Sin embargo, sí se hace un manejo adecuado de la Cratylia en su cultivo se puede reducir el contenido de la fibra Flóres et al., (2001).

Las ganancias de peso totales en los 25 días de experimentación para las dietas establecidas permiten observar que, a partir del momento de la inclusión de la cratilia en diferentes niveles en alimentación de los pollos, la que obtuvo un mejor resultado $(P<0.05)$ fue la dieta T0, (Tabla 3 y Gráfica 1 ), sin embargo, en el aumento diario de peso, fueron similares todos los tratamientos, la diferencia fue numérica y no estadística (Tabla 3). 
Aunque las diferencias de ganancia de peso se mantuvieron durante los cinco pesajes, favoreciendo a la dieta T0, se puede observar que a 20 días el peso del testigo era más bajo $(718 \mathrm{~g})$ en comparación con T1 $(750 \mathrm{~g})$ y T3 $(720 \mathrm{~g})$ teniendo en cuenta que la dieta T3 contenía un mayor porcentaje de Cratylia, después de los 25 días el testigo aventajo en peso de manera gradual a los demás tratamientos (Gráfica 1). Las conversiones alimenticias (consumo/ganancia de peso) fueron mayores $(\mathrm{P}<0.05)$ para T0 $(2.81)$ y $\mathrm{T} 1 \quad(3.20)$, presentando la conversión más baja T3, lo cual indica que el mayor contenido de fibra cruda y detergente neutro de esta dieta (Tabla 2), puede estar afectando esta variable, porque hay una disminución en su digestibilidad (52.1\%), lo cual afecta la disponibilidad de los nutrientes en el tracto digestivo (Tabla 3).

Tabla 3. Evaluación de pollo de engorde alimentado con diferentes porcentajes de Cratylia

\begin{tabular}{lcccc}
\hline \multirow{2}{*}{\multicolumn{1}{c}{ Variables }} & \multicolumn{4}{c}{ Tratamientos } \\
\cline { 2 - 5 } & T0 & T1 & T2 & T3 \\
\hline Días de experimentación & 25 & 25 & 25 & 25 \\
Peso inicial promedio (gr) & $445^{\mathrm{a}}$ & $450^{\mathrm{a}}$ & $440^{\mathrm{a}}$ & $442^{\mathrm{a}}$ \\
Peso final promedio (gr) & $1961,5^{\mathrm{c}}$ & $1817.1^{\mathrm{b}}$ & $1829.7^{\mathrm{b}}$ & $1705.8^{\mathrm{a}}$ \\
Aumento de peso total / ave (gr) & $1516,5^{\mathrm{c}}$ & $1367.1^{\mathrm{b}}$ & $1389.7^{\mathrm{b}}$ & $1263.8^{\mathrm{a}}$ \\
Aumento de peso / ave / día (gr) & $60.66^{\mathrm{a}}$ & $54.68^{\mathrm{a}}$ & $55.58^{\mathrm{a}}$ & $50.55^{\mathrm{a}}$ \\
Consumo de alimento/ ave/ día gr) & $171^{\mathrm{a}}$ & $175^{\mathrm{a}}$ & $189^{\mathrm{a}}$ & $182^{\mathrm{a}}$ \\
Consumo total alimento / ave (gr) & $4175^{\mathrm{a}}$ & $4375^{\mathrm{a}}$ & $4725^{\mathrm{a}}$ & $4550^{\mathrm{a}}$ \\
Conversión (consumo/peso) & $2.81^{\mathrm{a}}$ & $3.20^{\mathrm{a}}$ & $3.40^{\mathrm{b}}$ & $3.60^{\mathrm{c}}$ \\
Valor biológico (\%) & $84.4^{\mathrm{a}}$ & $83.5^{\mathrm{a}}$ & $80.1^{\mathrm{a}}$ & $78.9^{\mathrm{a}}$ \\
Digestibilidad FDN (\%) & $65.1^{\mathrm{b}}$ & $64.8^{\mathrm{b}}$ & $56.6^{\mathrm{a}}$ & $52.1^{\mathrm{a}}$ \\
Rendimiento en canal (\%) & $65.6^{\mathrm{c}}$ & $63.5^{\mathrm{bc}}$ & $58.5^{\mathrm{ab}}$ & $54.3^{\mathrm{a}}$ \\
Peso Pechuga (gr) & $432.9^{\mathrm{b}}$ & $422.8^{\mathrm{b}}$ & $414.8^{\mathrm{ab}}$ & $333.9^{\mathrm{a}}$ \\
Peso Pierna pernil (gr) & $351.1^{\mathrm{b}}$ & $269.3^{\mathrm{ab}}$ & $259.2^{\mathrm{a}}$ & $258.3^{\mathrm{a}}$ \\
Peso Ala con costillar (gr) & $150.4^{\mathrm{a}}$ & $149.6^{\mathrm{a}}$ & $147.1^{\mathrm{a}}$ & $146.1^{\mathrm{a}}$ \\
Peso rabadilla (gr) & $102.5^{\mathrm{a}}$ & $127.8^{\mathrm{a}}$ & $112.3^{\mathrm{a}}$ & $103.4^{\mathrm{a}}$ \\
\hline
\end{tabular}

Letras diferentes en la misma fila son distintas $(\mathrm{P}<0,05)$. Harina de Cratilya argentea $(\mathrm{HC})$. Testigo (T0) $=0 \%$ de $\mathrm{HC}, \mathrm{T} 1=5 \%$ de $\mathrm{HC}, \mathrm{T} 2=10 \%$ de $\mathrm{HC}$ y $\mathrm{T} 3=15 \%$ de $\mathrm{HC}$.

En la relación de peso final promedio de las aves y el peso total de la canal y pierna pernil, las dietas que obtuvieron un mejor resultado fueron T0 y T1 ( $\mathrm{P}<0.05)$, (Tabla 3, Gráficas 2 y 3). Sin embargo, en el pesaje de la pechuga T2 
fue similar a estos dos tratamientos. El peso del ala con costillar y rabadilla fueron similares para las cuatro raciones suministradas a los pollos. Es de anotar que, en el valor biológico de la proteína, todos los tratamientos fueron similares, y se puede evidenciar la alta calidad de la proteína de Cratylia, puesto que su inclusión hasta en el $15 \%$ en la dieta para los pollos no influyó con relación al testigo, cuyas fuentes de proteína se basaron en la harina de carne y torta de soya únicamente.

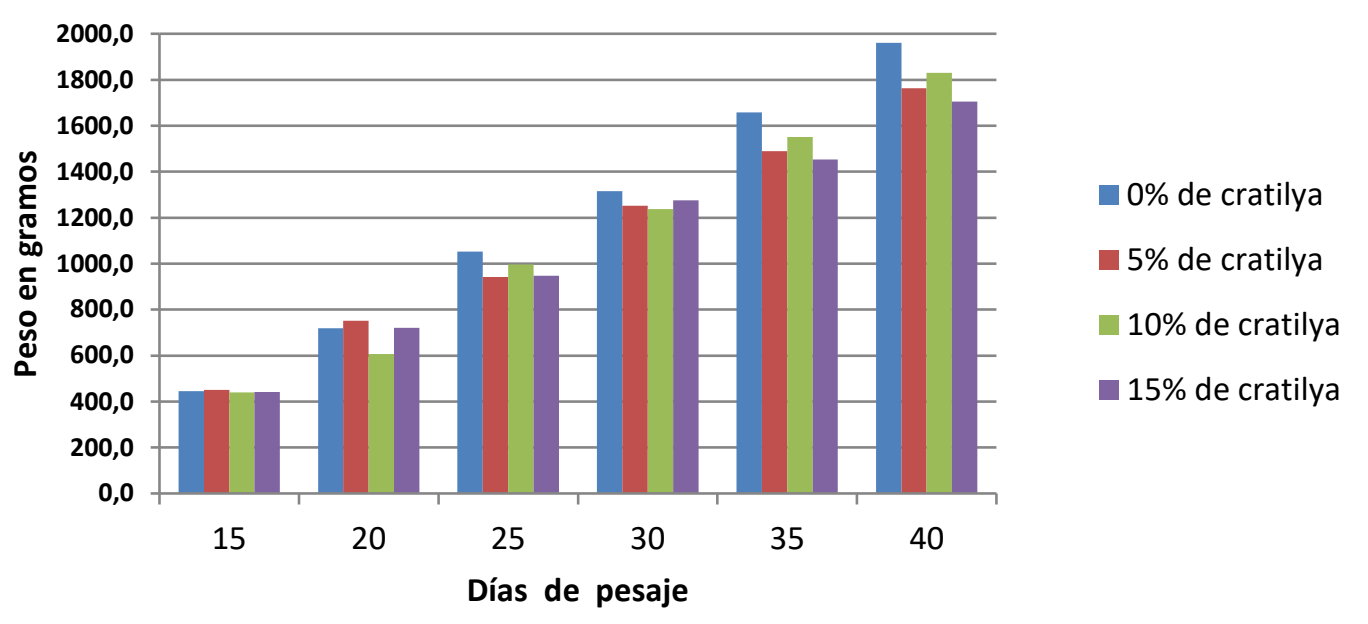

Gráfica 1. Aumento de peso (g) de los pollos durante 25 días

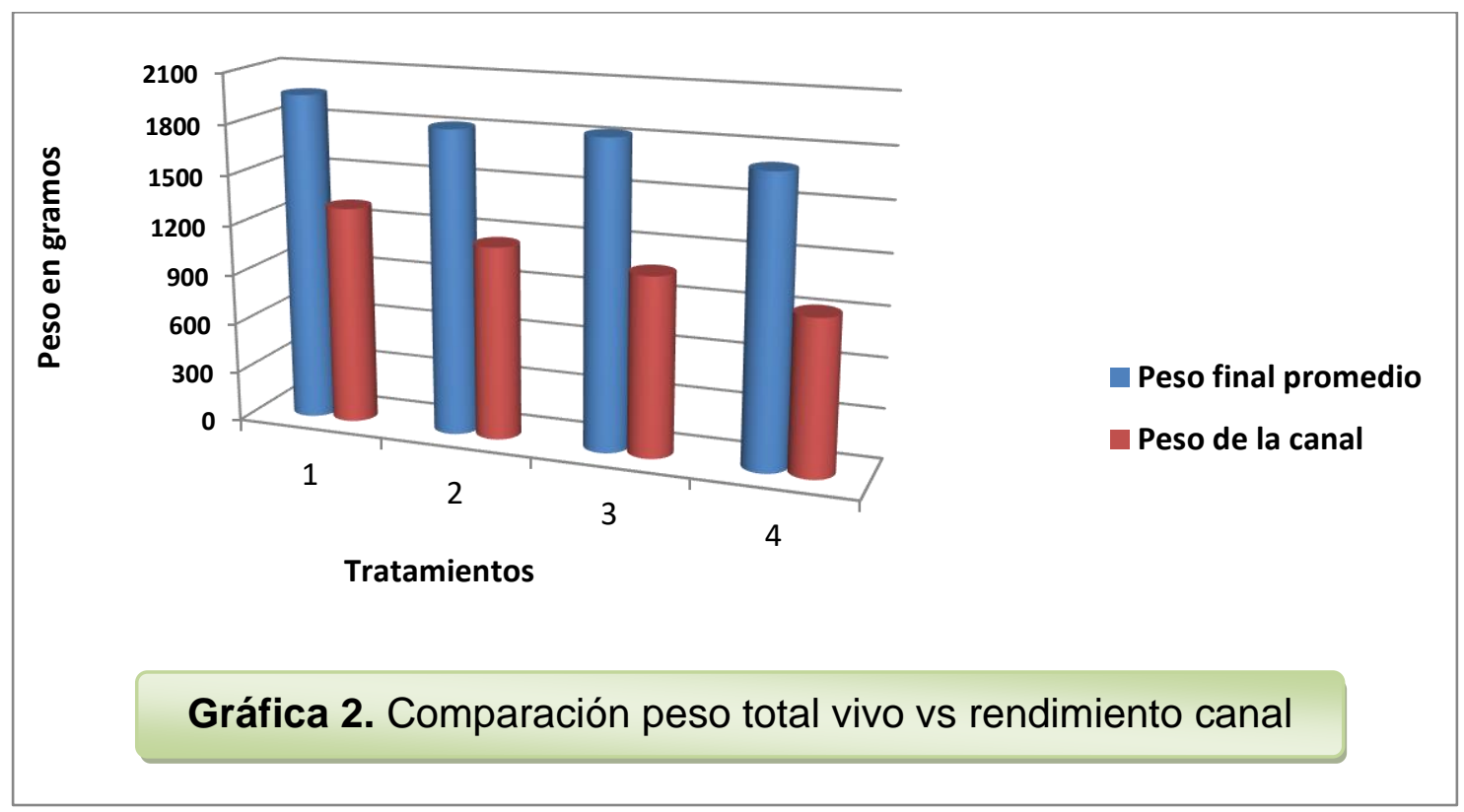


Estos resultados concuerdan con los de Roa, (2000) cuando incluyó en dietas para pollos de engorde $12 \%$ de harina de la arbustiva forrajera cayeno (Hibiscus rosa-sinensis), y no se afectó negativamente el valor biológico de la proteína y en este caso tampoco la digestibilidad de la fibra detergente neutro se redujo, lo cual fue diferente en este experimento porque, la digestibilidad de la fibra si disminuyó cuando se reemplazó con el 10\% de Cratylia en las dietas de los pollos.

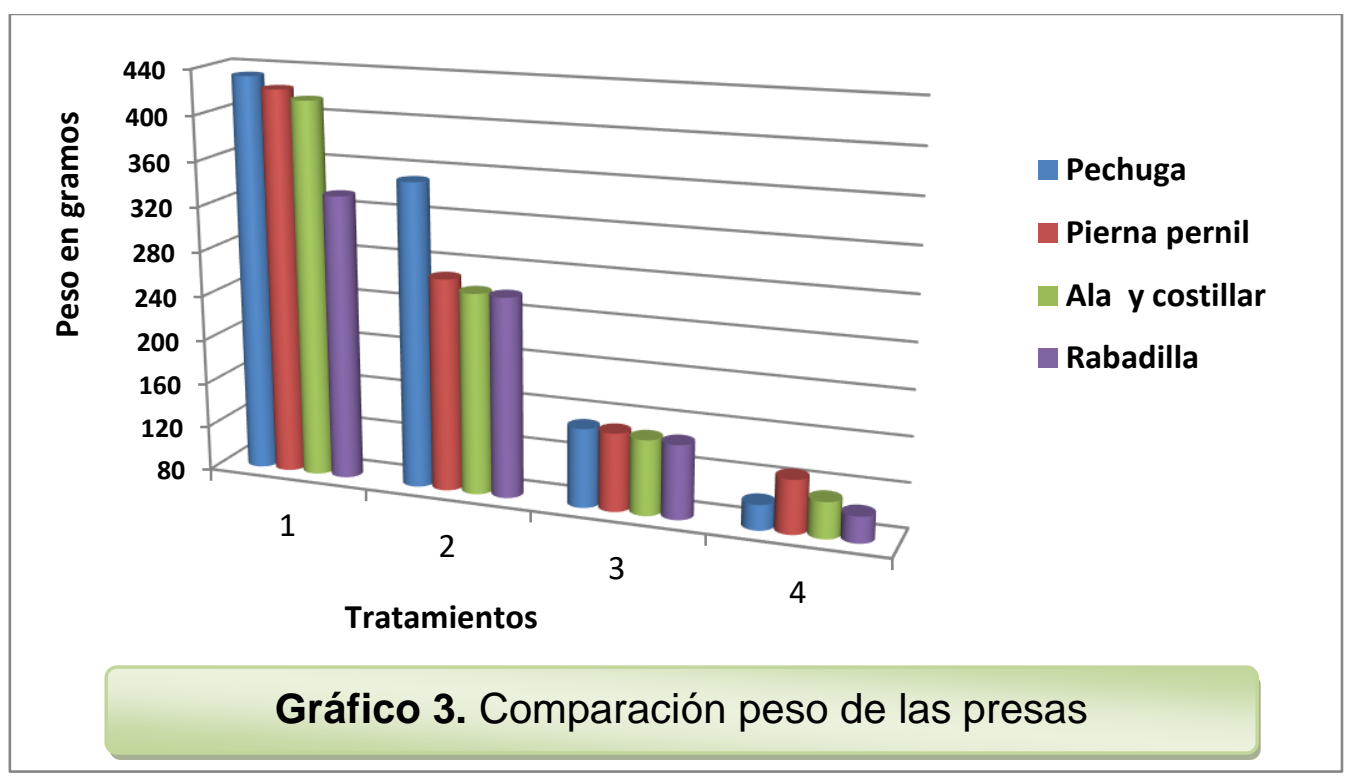

\section{CONCLUSIONES}

Los pollos que obtuvieron una mejor ganancia de peso total fueron los alimentados con las dietas T0 y T2, al igual que el peso total de la canal, sin embargo, al momento del pesaje de las presas se observó que la pierna pernil fue similar para estos dos tratamientos, pero es importante anotar, que el peso de la pechuga de T2 fue similar a T0 y T1. De acuerdo a estos resultados se deduce que la inclusión del 5\% harina de Cratylia en dietas para pollos de engorde no afecta estas variables.

El porcentaje de valor biológico de la proteína, que mide la calidad de este nutriente, fue similar para los cuatro tratamientos, aunque la fibra que contiene la Cratylia, afectó la digestibilidad de la fibra detergente neutro, la cual decreció a 
medida que se incrementaron los niveles de esta leguminosa en la dieta para los pollos de engorde.

\section{BIBLIOGRAFÍA}

1. Abreu A., Carulla J., Lascano C., Díaz E., Kreuzer M., Cano T. Efecto del fruto, del pericarpio y del extracto semipurificado de saponinas de Sapindus saponaria sobre la fermentación y la metanogénesis in vitro en un sistema RUSITEC. Revista Colombiana de Ciencias Pecuarias, 16 (2):147-154. 2003

2. AOAC. Official Methods of Analysis (18 $\left.{ }^{\text {th }}\right)$. Association of Official Analytical Chemists, Arligton, VA. Washington, D.C. 2005.

3. Flores O. I., Bolívar M., Botero J., Ibrahim M. Parámetros nutricionales de algunas arbóreas leguminosas y no leguminosas con potencial forrajera para la suplementación de rumiantes en el trópico. Centro Agronómico Tropical De Investigación y Enseñanza, Turrialba, Costa Rica. 2001.

4. Franco M. H., Muhammad I., Pezo D., Camero A., Araya J. Degradabilidad ruminal in situ y solubilidad de la proteína de rebrotes de cratilia de diferentes edades. 2000.

5. Jiménez A., Tovar M. Evaluación de la producción de materia seca y valor nutritivo de (Cratylia argentea (desv.) O. Kuntze) a dos edades y dos alturas de corte en época seca y de lluvias en la finca Altamonte, municipio de Sahagún, departamento de Córdoba Tesis pregrado. Facultad Ciencias Agropecuarias. Universidad de Sucre. 48 p. 2003.

6. Lascano C., Rincón A., Plazas C., Ávila P., Bueno G., Argel P. J. Cultivar Veranera (Cratylia argentea (Desvaux O. Kuntze). Leguminosa arbustiva de usos múltiples para zonas con períodos prolongados de sequía en Colombia. CORPOICA, CIAT, Ministerio de Agricultura y Desarrollo Rural de Colombia. $28 \mathrm{p}$.

7. Lascano C., Plazas C. Utilidad de la leguminosa semiarbustiva Cratylia argentea en sistemas de ganado doble propósito del Piedemonte Llanero. Validation y difusion. Código 201504059. Informe Final. 1. 2003.

8. Miles J., do Valle C. V, Rao I. M., Euclides V. P. B. Brachiaria grasses. Warm season (C4): grasses \& agronomy. Monograph No 45. American Society of Agronomy, Crop Science Society of America, Soil Science Society of America (EUA). p. 745-783. 2004

9. Plazas C. H. Estrategias de suplementación con el uso de Cratylia argentea cv. Veranera, en los Llanos Orientales de Colombia. Revista Sistemas Agroecológicos. Editado por María Ligia Roa. Grupo de Agroforestería UNILLANOS. Diciembre de 2009. Recuperado 25 Junio 2011. www.sistemasagroecologicos.com

10. Rincón A. Ceba de bovinos en pasturas de Brachiaria decumbens suplementados con caña de azúcar y Cratylia argentea. Pasturas tropicales. CORPOICA. 2005.

11. Reyes H. M. Análisis económico de experimentos agrícolas con presupuestos parciales: Re-Enseñando el uso de este enfoque. Boletín Informativo, Centro 
de Información Agrosocioeconómica, Universidad San Carlos de Guatemala. 2001.

12. Roa M. L. Producción de pollos de engorde alimentados con harina de cayeno (Hibiscus rosa-sinensis) En: Informe técnico, de proyecto de investigación Utilización de árboles forrajeros para la alimentación animal: Roa M. L., Sanabria D., Galeano J., Muñoz R. y Muñoz J. Convenio UNILLANOS COLCIENCIAS, Villavicencio, Colombia. 800 p. 2001.

13. Roa M. L. Pollos alimentados con diferentes niveles de harina de Trichanthera gigantea y Erythrina poeppigiana. Rev. Sist. Prod. Agroecol. 1, (2): 22-33. 2010.

14. Romero R., González J. Efecto de la alimentación durante la época seca con Cratylia argentea, fresca y ensilada sobre la producción de leche y sus componentes. Documento No 184, CIAT, Cali, Colombia. p 15-18. 2004.

15. Seddon, I. El uso de sustancias alimentarias alternativas en las dietas porcinas (en línea). Artículo Técnico, Foro de Porcicultura. Comunidad de Negocios Internacionales Relacionados con la Producción Animal. 2004. Disponible En: http://www.engormix.com/MA-porcicultura/nutricion/articulos/uso-sustanciasalimentarias-alternativas-t323/141-p0.htm 